\title{
Bad Obstetric History and Infectious Causes
}

\author{
Rema Devi, N. Sreenivas, Sayee Rajangam \\ Division of Human Genetics, Department of Anatomy, St. John's Medical College, \\ Bangalore -560 034, Karnataka, India .
}

\section{INTRODUCTION}

Bad Obstetric History (BOH), a topic with variable definitions, has been studied with different standardizations for various parameters like epidemiology, etiology and management. It is well realized that at least $12-15 \%$ of all recognized conceptions end in miscarriage, and pre-clinical pregnancy loss rate is still higher $-22-30 \%$. At present, there is no hard evidence that bacterial or viral infections can cause recurrent abortions. An impressive incidence of antichlamydial antibody has been reported in women with 3 or more spontaneous abortions, but it is not certain whether this is associated with Chlamydia trachomatis or whether this is a marker of different immune response in women with recurrent abortions. Other organisms that have been implicated include Toxoplasma gondii, Listeria monocytogenes, Mycoplasma homonis, Herpes virus and Cytomegalo virus. Claims of effective antibiotic treatment have been derived without benefit of randomized studies. (Paul 1994).

Families seek and deserve answers regarding the cause of the loss of a baby and they are eager to know the risk of recurrence. The goal in evaluation of a pregnancy loss is to provide families with an accurate diagnosis and information on which to base the future pregnancy planning and management.

The present study attempts to identify the role of infectious agents in repeated pregnancy wastage.

\section{MATERIALSAND METHODS}

104 couples with a bad obstetric history referred to the Division of Human Genetics, St. John's Medical College for cytogenetic investigations and genetic counseling were taken up for the study. The patients were mainly those who were referred from St. John's Medical College Hospital, nursing homes and other hospi- tals in and around Bangalore and mainly comprised of patient population from the south Indian states of Karnataka, Kerala, Tamil Nadu and Andhra. Couples were taken for study only if they had two or more than two abortions or neonatal deaths or off-springs with multiple congenital anomalies. A detailed proforma, which included suspected causal factors, especially infectious and environmental factors were obtained from the couples. All relevant investigations were asked for so as to ascertain a probable cause for their presenting problem. The woman was checked for TORCH IgG and IgM antibodies and the couple was ask, to have blood VDRL assessed. The association of these factors with the etiology of $\mathrm{BOH}$ was done with relevant statistical analysis. Once the causal factor was identified, appropriate genetic counseling was given with regard to recurrence risk, management and subsequent follow-up.

\section{RESULTS AND DISCUSSION}

Out of the 104 couples studied, 20 females showed evidence of TORCH infection. Their age ranged from $20-37$ years. The number of abortions ranged from 2-8. There was no family history of similar problems. ( Table 1 ).

Even though there are various reports that specific infectious agents cause recurrent abortions, there is no concrete evidence as to the role of specific bacterial or viral agents in recurrent abortions. Even though Chlamydia has been found to be associated with repeated abortions, its exact role is not clear-cut. (Paul 1994). Summers (1994) suggested that infection is an occasional cause of sporadic spontaneous abortion and consistent with statistical probability, recurrent miscarriage due to infection occurs with a frequency that is much low. In the medical literature, the limited evidence linking infection and recurrent pregnancy loss in humans remains largely anecdotal and generally cannot be reproduced in prospective studies. The role of 
Table I: BOH And Infectious Causes (TORCH)

\begin{tabular}{lcc}
\hline \hline Serial No. & $\begin{array}{c}\text { Maternal } \\
\text { Age }\end{array}$ & $\begin{array}{c}\text { No. of } \\
\text { Abortions }\end{array}$ \\
\hline \hline 1 & 21 & 3 \\
2 & 29 & 2 \\
3 & 28 & 3 \\
4 & 29 & 6 \\
5 & 25 & 2 \\
6 & 37 & 4 \\
7 & 36 & 6 \\
8 & 20 & 3 \\
9 & 25 & 3 \\
10 & 24 & 2 \\
11 & 26 & 4 \\
12 & 25 & 2 \\
13 & 21 & 4 \\
14 & 21 & 3 \\
15 & 34 & 4 \\
16 & 37 & 3 \\
17 & 26 & 3 \\
18 & 27 & 8 \\
19 & 23 & $3.30 \pm 1.66$ \\
20 & 28 & p 0.013 \\
\hline \hline Mean & 27.11 & \\
\hline \hline Significant & & \\
\hline
\end{tabular}

maternal and fetal infections in the recurrent $1 \mathrm{st}$ trimester loss remains controversial. This reflects the low frequency with which the necessary microbial and maternal factors combine to cause recurrent miscarriage by infection of the fetal tissue or by stimulation of an Antigen - Antibody response. The patient's susceptibility to chronic infection must play a determining role in some of the reported cases. Probable factors that play a role in the risk of abortion due to infection are:

i) either due to primary exposure during early gestation to organisms like Toxoplasma gondii, Rubella, Chlamydia trachomatis and Cytomegalo virus and the capability of the organism to cause placental infection and development of an infectious carrier state.

ii) due to immunocompromised women if the Immmunoglobin titre is not normal.

iii) due to immunosuppressants, chemotherapy, corticosteroids, or acquired immune deficiency syndrome.

Exposure to a microbe, which could establish a chronic infection and spread to the placenta in an immunocompromised patient, is probably the most obvious risk situation for habitual abortion. In routine medical practice, it is not necessary or efficient to screen universally for the unexpected, but it is necessary to be aware of the rare possibilities. Most patients with a history of recurrent miscarriage will not benefit from an extensive infection workup. Rae et al. (1994) concluded that there is no association between immunoglobulin G antibodies to Chlamydia trachomatis and recurrent spontaneous abortion. Zavala-Velazquez et al. (1989) analysed 100 cases and found $47 \%$ of them to have antibodies against Toxoplasma. Galvan Ramirez et al. (1995) found that seroprevalence of toxoplasma varie from $7 \%$ to $51.3 \%$ in pregnant women, and in women with abnormal pregnancies and abortions the seroprevalence varie from $17.5 \%$ to $52.3 \%$. Cauchi et al. (1991) examined 165 women with a history of three or more consecutive miscarriages in the first trimester for factors that may have a bearing on subsequent pregnancy success or failure and found that factors that were found to correlate significantly with success rate were length of abortion history, total number of abortions, interval from last miscarriage to present pregnancy, and degree of subfertility. The uterine endometrium undergoes changes and the chances for a perfect implantation is less.

In the present study, these organisms have been implicated, Toxoplasma gondii, Rubella, Chlamydia trachomatis and cytomegalo virus and 20 women with Toxoplasma, Rubella, Chlaymidia, and Herpes infection had a frequency of $3.30 \pm 1.66$ abortions. This was found to be significant $\mathrm{p}<0.05$. These organisms produce toxic metabolic by products, endotoxin, exotoxin, or cytokines, which have a direct or indirect effect on the uterus or the feto-placental barrier, resulting in $\mathrm{BOH}$.

Counseling was given to the patients in the form of further treatment whenever necessary, and subsequent risk of recurrence.

\section{CONCLUSION}

Bad Obstetric History could be because of environmental factors: infections, radiation, occupational hazards, addictions and habits. This study has confirmed the significant association of infectious causes, especially TORCH, and $\mathrm{BOH}$. TORCH infections are considered to be a known causal factor, which is treatable.

KEY WORDS Recurrent abortions; TORCH; etiology; counseling

ABSTRACT This article refers to the association be- 
tween Bad Obstetrics History (BOH) and infectious causes. Data has been gathered from 104 consecutively referred couples with bad obstetrics history for karyotyping and genetic counseling to the Division of Human Cytogenetics, St. John's hospital. It was found that 20 women, aged 21 - 37 years, had raised IgG antibody titer against TORCH infections. These women had $2-8$ abortions; mean was $3.30 \pm 1.66$ ( p 0.013); their karyotypes were normal. The association between $\mathrm{BOH}$ and infection was found to be significant. During genetic counseling, the couples were explained the nature of their problem and the chances of having a successful pregnancy, and the female partners were referred to gynecologists for treatment.

\section{REFERENCES}

Cauchi MN, Pepperell R, Kloss M, Lim D 1991.

Predictors of pregnancy success in repeated miscarriage. Am J Reprod Immunol, 26(2): 72-5.

Galvan Ramirez M de la L, Soto Mancilla JL, Velasco Castrejon O, Perez Medina R 1995. Incidence of anti-Toxoplasma antibodies in women with highrisk pregnancy and habitual abortions 1995. Rev Soc Bras Med Trop, 28(4): 333-7.

Paul ME 1994. Disorders of reproduction. Prim Care, 21(2): $367-86$

Rae R, Smith IW, Liston WA, Kilpatrick DC 1994. Chlamydial serologic studies and recurrent spontaneous abortion. Am J Obstet Gynecol, 170(3): 782-5.

Summers PR 1994. Microbiology relevant to recurrent miscarriage. Indian J Med Res, 100:19-22.

Zavala-Velazquez J, Guzman-Marin E, Barrera-Perez M, Rodriguez-Felix ME 1989. Toxoplasmosis and abortion in patients at the O'Horan Hospital of Merida, Yucatan. Akush Ginekol (Mosk), 6: 20-3. 